\title{
Instituições de Longa Permanência para Idosos no Brasil: revisão integrativa da literatura
}

Long-stay Institutions in Brasil: an integrative literature review

Instituciones de largo plazo para ancianos en Brasil: una revisión integradora de literatura

Patricia do Nascimento Tavares Olga Susana Costa Couto e Araújo Josiane Ferreira de Mello Andreza de Nóvoa Rocha Maciel

Maria José D’Elboux

RESUMO: Objetivou-se levantar e analisar a produção científica acerca do delineamento dos trabalhos publicados e temas estudados sobre as instituições de longa permanência para idosos no Brasil, produção essa arrolada no LILACS, MEDLINE, BDENF e SciELO no período de 2013 a 2017. O corpus constitui-se por 88 artigos. Nota-se um número insipiente de pesquisas com intervenções, visto que os pesquisadores ainda buscam descrever o perfil dos idosos institucionalizados, segundo critérios de saúde e doença.

Palavras-chave: Serviços de Saúde para Idosos; Idoso; Instituição de Longa Permanência para Idosos.

ABSTRACT: It was aimed to review the literature about Brazilian nursing homes for seniors published at LILACS, MEDLINE, BDENF and SciELO between 2013 and 2017. The corpus consists of 90 articles. There is an incipient amount of papers about intervention research, as long as the researchers still seek to describe the profile of the resident seniors, according to health and disease criteria.

Keywords: Health Services for the Aged; Aged; Homes for the Aged. 
RESUMEN: El objetivo fue encuestar y analizar la producción científica sobre la delineación de los trabajos publicados y los temas estudiados sobre las instituciones de atención a largo plazo para ancianos en Brasil. El corpus consta de 88 artículos. Existe un número incipiente de investigaciones con intervenciones, ya que los investigadores todavía buscan describir el perfil de los ancianos institucionalizados, de acuerdo con los criterios de salud y enfermedad.

Palabras clave: Servicios de salud para ancianos; Viejo hombre; Institución de atención a largo plazo para ancianos.

\section{Introdução}

De acordo com o Regulamento Técnico para o Funcionamento das Instituições de Longa Permanência para Idosos (ILPIs), constante na Resolução da Diretoria Colegiada, a RDC 283, de 26 de setembro de 2005, da Agência Nacional de Vigilância Sanitária, as ILPIs podem ser denominadas como instituições governamentais ou não governamentais, de caráter residencial, destinadas a domicílio coletivo de pessoas com idade igual ou superior a 60 anos, com ou sem suporte familiar, em condições de liberdade e dignidade e cidadania (Brasil, 2005).

As ILPIs são instituições que prestam assistência aos idosos, garantindo condições de bem-estar físico, emocional e social, em conformidade, entre outros, com o Estatuto do Idoso, com a legislação vigente e com as políticas públicas relacionadas a essa população (Born, \& Boechat, 2013; Camarano, \& Kanso, 2010).

Essas instituições surgiram no Brasil na década de 1980 e foram os primeiros locais destinados a cuidar da saúde dos idosos e a suprir suas necessidades básicas, como alimentação e moradia (Pestana, \& Espírito Santo, 2008). Em virtude desse cenário, o Ministério da Saúde, em 1989, publicou a Portaria n. ${ }^{\circ}$ 810/1989, a qual foi a primeira a definir as Normas e Padrões de Funcionamento de Casas de Repouso, Clínicas Geriátricas e outras instituições para idosos, quando se definia como deveria ser a organização da instituição, áreas físicas, instalações e os recursos humanos (Brasil, 1989). 
A Portaria MS 810/89, até maio de 2001, quando foi assinada a Portaria SAS 073/01, era o único instrumento legal para criar normas para as instituições (Born, \& Boechat, 2013).

O número de instituições destinadas ao acolhimento de idosos nas formas de moradia em nosso país começou a crescer a partir das últimas décadas do século XX, sendo tal crescimento decorrente às demandas de uma sociedade que aumenta a expectativa de vida e diminui a disponibilidade de recursos familiares para o cuidado dos idosos (Camarano, \& Kanso, 2010).

Observa-se, na literatura sobre o tema, estudos relacionados aos residentes da ILPIs em sua maioria (Silva, Dias, \& Piazza, 2017; Scarabottolo, et al., 2017; Hartmann Junior, \& Gomes, 2017; Lini, Portella, \& Doring, 2016), concentrando-se em aspectos médicos e clínicos dos idosos e frequentemente com discussões sobre as demandas assistenciais (Roquete, Batista, \& Arantes, 2017). Entretanto, após análise das questões abordadas, denotase a necessidade de mapear os estudos disponíveis sobre ILPIs, com vistas a delinear o estado da arte dos problemas emergentes. Uma das dimensões mais relevantes para a compreensão do fenômeno da inserção do idoso no contexto institucional diz respeito aos motivos que deflagram a busca das ILPIs (Alves-Silva, Scorsolini-Comin, \& Santos, 2013).

Em consonância a essas considerações, Roquete, et al. (2017) concluem, em seu estudo, a necessidade de investimentos em pesquisas descritivas sobre as ILPIs no Brasil, a fim de que o conhecimento teórico desenvolvido possa fomentar ações positivas nas práticas dessas organizações. Os autores ressaltam a escassez de trabalhos que abordem mais especificamente, e de forma aprofundada, as demandas gerenciais das ILPIs, o que possibilitaria conhecer práticas organizacionais adotadas por essas instituições, bem como avanços e desafios no âmbito da gestão.

Tendo em vista a importância desta temática, indaga-se sobre qual enfoque tem sido dado na literatura sobre as ILPIs no Brasil nos últimos anos, a fim de se identificar lacunas e obter contribuições para o direcionamento de novas pesquisas e políticas direcionadas às instituições, seus usuários e recursos humanos. Nesta perspectiva, o presente estudo tem o objetivo de buscar o delineamento dos trabalhos publicados e os temas abordados sobre o tema das ILPIs brasileiras em trabalhos realizados em âmbito nacional, no período de janeiro de 2013 e outubro de 2017. 


\section{Método}

A presente pesquisa foi realizada por meio de revisão integrativa da literatura que, de acordo com Whittemore e Knaf (2005), permite a apreciação da bibliografia e ainda ativa a reflexão sobre a necessidade de futuros estudos. Foram adotadas as etapas preconizadas por esses autores: formulação de problema, elaboração de critérios para inclusão e exclusão de estudos (amostragem), pesquisa na literatura, análise e categorização dos estudos e apresentação da síntese do conhecimento. A pergunta norteadora adotada neste estudo foi: Qual é o delineamento dos trabalhos publicados e quais são os temas estudados sobre as ILPIs?

A busca de artigos foi realizada nas bases de dados Medical Literature Analysis and Retrieval System Online (MEDLINE), Literatura Latino Americana em Ciências da Saúde (LILACS), Banco de Dados em Enfermagem (BDENF) e Scientific Eletronic Library Online (SciELO). O período selecionado foram os últimos cinco anos compreendendo janeiro de 2013 a outubro de 2017.

Para seleção dos estudos, utilizaram-se os descritores: "institucionalização", “instituição de longa permanência", "asilo", “idosos” e seus correlatos em inglês. Para compor o corpus de análise da presente revisão, foram incluídos apenas artigos empíricos, com texto completo disponível, publicados em língua portuguesa, inglesa e espanhola, e desenvolvidos no Brasil, não se fazendo restrição quanto ao perfil da amostra, sendo incluídos estudos realizados com idosos residentes e profissionais que atuavam em ILPIs. Foram excluídos artigos de revisão e análise documental de prontuários, dissertações e teses, bem como os estudos que não tinham relação direta com a temática.

Inicialmente, foram localizados 212 artigos nas bases de dados MEDLINE, LILACS, BDENF e SciELO, a partir dos descritores utilizados e critérios de inclusão definidos. Logo após, procedeu-se a leitura dos resumos, e exclusão dos artigos que não mantinham relação direta com a temática do estudo, e artigos duplicados nas bases de dados, resultando em 88 artigos. Dada a limitação de espaço, os estudos localizados não serão listados; todavia, a relação poderá ser obtida junto à primeira autora.

Na sequência, procedeu-se a leitura exaustiva dos estudos selecionados, cujos dados de interesse foram tabulados em planilha eletrônica, segundo os objetivos do estudo propostos, permitindo análise e discussão dos resultados. 
Os estudos foram analisados segundo os seguintes recortes organizacionais: delineamento das pesquisas, incluindo-se: 1) Delineamento das publicações; 2) perfil dos participantes dos estudos; 3) instrumentos utilizados para coleta dos dados; 4) área de conhecimento dos autores; e 5) principais temáticas abordadas.

\section{Resultados}

A amostra conta com 88 publicações que foram analisadas de acordo com o delineamento dos estudos e as temáticas abordadas.

\section{Delineamento das publicações}

Destas pesquisas, a maioria $(n=78)$ tem amostras constituídas por estudos com foco em idosos. Quanto ao perfil dos participantes dos estudos, observa-se na Tabela 1, que as amostras foram constituídas, em sua maioria, por sujeitos de ambos os sexos; entretanto, em 4 estudos $(5,13 \%)$, as amostras eram exclusivamente femininas. Este fato pode ser justificado pelo predomínio de mulheres nas ILPIs e a sobrevida maior que a dos homens, conforme destacado pela literatura (Roquete, et al., 2017).

Tabela 1 - Perfil dos idosos participantes dos estudos (n=78). Campínas, SP, 2018

\begin{tabular}{lccc}
\hline Variável & $\mathrm{N}$ & $\%$ & Amplitude \\
\hline Gênero & & & \\
\hline Ambos os sexos & 40 & 51,28 & \\
Feminino & 44 & 43,59 & \\
Não Especificado & & & $6-1192$ indivíduos \\
\hline Tamanho Amostral & 51 & 65,38 & \\
\hline Até 100 indivíduos & 15 & 19,23 & \\
101 a 200 indivíduos & 12 & 15,38 & $60-113$ anos \\
Acima de 200 & & & \\
indivíduos & 29 & 37,18 & \\
\hline Faixa Etária & 14 & 17,95 & $1-13$ anos \\
\hline Até 80 anos & 35 & 44,87 & \\
Acima de 80 anos & & & \\
Não Informado & 6 & 8,69 & \\
\hline Tempo de Institucionalização & 7 & 83,33 & \\
\hline Até 5 anos & 65 &
\end{tabular}


O tamanho das amostras apresentou grande variabilidade, desde 6 até 1192 sujeitos, sendo que a maioria $(65,38 \%)$ contou com até 100 idosos. Apenas $15,38 \%$ dos estudos têm mais de 201 indivíduos na amostra; constatou-se que estudos com abordagem qualitativa contam com amostras inferiores a dez sujeitos.

Analisando grupos etários dos inquiridos nos estudos, nota-se que 44,87\% não especificam as idades médias das amostras; porém, apurou-se que 37,18\% contemplam indivíduos acima dos 60 anos, idade regulamentada pela Organização Mundial da Saúde, OMS (Brasil, 2005) para os países em desenvolvimento ou emergentes. Sabe-se que internacionalmente se utiliza, para pesquisa, idades acima de 65 anos; na revisão percebe-se que as idades surgem escalonadas por conveniência das próprias amostras. Uma parte considerável analisa cidadãos acima dos 80 (17,95\%), demanda que surge com pesquisa de cidadãos mais longevos. O tempo de institucionalização foi citado em apenas 13 artigos; a maioria dos artigos $(83,33 \%)$ não refere esta variável.

Importante salientar que, em 26 estudos, os dados foram coletados em mais de uma ILPI, para compor a amostra de forma mais robusta ou por conveniência de estudo. Por último, apenas 5,13\% dos artigos utilizam amostras que abordam conjuntamente indivíduos institucionalizados e da comunidade (sem estarem em instituições de longa permanência), sendo apenas estudos descritivos, e sem contemplarem a interação dos residentes das ILPIs e a comunidade. Pesquisas com enfoque na interação poderão surgir, tendo em conta que a própria regulamentação da ANVISA (DOU n. ${ }^{\circ}$ 186, de 27 setembro de 2005) refere esta necessidade. Na presente revisão, 11,11\% $(\mathrm{n}=10)$ abordaram a regulamentação dos recursos humanos nas ILPIs de forma descritiva, sem análise de intervenções ou recorte comparativo.

Dos artigos arrolados, cuja amostra foi composta por profissionais, ocupacionais e estagiários, $50 \%(\mathrm{n}=5)$ deles deram enfoque à equipe de enfermagem; 30\% $(\mathrm{n}=3)$ trabalharam com a equipe multiprofissional; $10 \%(\mathrm{n}=1)$ tratava de cuidadores; e 10\% $(\mathrm{n}=1)$ discutia sobre estagiários de enfermagem.

Com relação ao tipo de pesquisa, todos os estudos eram observacionais e descritivos ( $n=88$ ), com exceção de dois estudos de ensaio clínico, sendo que 80\% eram estudos com corte transversal; e 4\%, longitudinal. Trabalhos do tipo exploratório, que possibilitam uma aproximação do pesquisador com o tema de interesse, corresponderam a $16 \%$ da amostra. Quanto ao tipo de abordagem, 60\% dos estudos foi quantitativa; 23\% qualitativa; enquanto em $10 \%$ foram empregadas ambas as abordagens. E para os estudos cujo desenvolvimento é o tempo, $4 \%$ dos artigos foram considerados prospectivos e $3 \%$, retrospectivos. 
Quanto ao perfil dos profissionais, nos estudos, observa-se que as amostras são constituídas, em sua maioria, também por sujeitos de ambos os sexos, não havendo no rol de artigos, abordagem de somente profissionais do sexo masculino. Os estudos realizados com profissionais, em sua maioria, visam a identificar os eventos potencialmente estressores relacionados à prática profissional, e o impacto psicológico aos trabalhadores (Mariano, \& Carreira, 2016a; Mariano, \& Carreira, 2016b; Mariano, Baldissera, Martins, \& Carreira, 2015; Salcher, Portella, \& Scortegagna, 2015).

Outro aspecto investigado nos estudos realizados com os profissionais que atuam em ILPIs refere-se às estratégias de trabalho utilizadas pelos mesmos, para dirimir conflitos entre os residentes (Bruinsma, et al., 2017), bem como otimizar os cuidados oferecidos (Clos, \& Grossi, 2016; Mariano, et al., 2015).

A percepção do cuidado humano relativamente à pessoa idosa institucionalizada foi objeto de estudo de três pesquisas levantadas (Medeiros, Felix, \& Nobrega, 2016; Medeiros, Oliveira, Lima, \& Nobrega, 2015; Nunes, Nunes, Marinho, \& Fernandes, 2014). Apenas um dos estudos investigou o conhecimento dos profissionais da área da saúde acerca da Comunicação Suplementar e Alternativas (CSA), estratégia utilizada para minimizar prejuízos comunicativos e sociais entre idosos com disfunção neurolinguística (Comiotto, Kappaun, \& Cesa, 2016).

No que diz respeito aos instrumentos utilizados para a coleta de dados, verifica-se que em 24,44\% dos estudos $(n=22)$, foi empregado um questionário para coleta de dados sociodemográficos e informações de saúde, enquanto a entrevista semiestruturada foi aplicada em 14,44\% $(\mathrm{n}=13)$ dos estudos. Também foram realizadas análises bioquímicas (Pfrimer, et al., 2015), análises parasitológicas e microbiológicas (Torres, et al., 2016) e aferição de medidas antropométricas (Volpini, \& Frangella, 2013; Negreiros, Cavalcanti, Marques, Ricardo, \& Fontenele, 2014; Borges, Silva, Clares, Nogueira, \& Freitas, 2015; Rocha, et al., 2016).

Especificamente para a avaliação do idoso, foram identificados 28 instrumentos distintos nos estudos levantados. Observa-se um predomínio dos instrumentos que avaliam cognição, em 29,11\% dos estudos. Os instrumentos amplamente utilizados pelos pesquisadores para avaliação de funções cognitivas foram o Mini-Exame do Estado Mental ( $n=17)$; Teste de Fluência Verbal e Teste do Desenho do Relógio ( $n=2)$, cada; e Teste de Trilhas $(\mathrm{n}=1)$. 
A capacidade funcional foi investigada em $26,58 \%$ dos estudos, por meio da Escala de Lawton (n=5), Escala de Pfeiffer (n=3), Índice de Bathel (n=2), Índice de Katz (n=9) e Escala de Avaliação da Incapacidade Funcional para Demência $(n=1)$. Verifica-se, ainda, um interesse por parte dos pesquisadores em investigar equilíbrio e quedas (22,78\%), sendo a Escala de Equilíbrio de Berg $(n=8)$, e o TimedUp and Go $(n=7)$, os instrumentos mais prevalentes nas amostras estudadas. A presença de sintomas psiquiátricos, em especial, a depressão, foi avaliada em 11,39\% dos estudos, seguida da avaliação da qualidade de vida e lazer, com 10,13\%. Nessas categorias, utilizou-se a Escala de Depressão Geriátrica de Yesavage e o WHOQOL, respectivamente, na maioria dos estudos levantados.

Os dados levantados corroboram evidências disponíveis na literatura especializada, que indicam que o comprometimento de ordem cognitiva e funcional são fatores de institucionalização do idoso, visto que os idosos com maior idade permanecem em seus domicílios na ausência de incapacidades importantes (Lini, et al., 2016). Em congruência ao exposto, Trindade, Barboza e Borges (2013) destacam que idosos institucionalizados apresentam menor desempenho cognitivo, repercutindo, negativamente, nas habilidades funcionais e no aumento de seus sintomas depressivos.

Em recente estudo de revisão realizado por Barros, Santos, Gonzaga, Lisboa e Brand (2016), verificou-se que diversos fatores relacionados à capacidade funcional de idosos institucionalizados, dentre eles, a cognição, a qualidade de vida e a aptidão física. Os autores encontraram maior incidência de quedas, dependência para atividades básicas de vida diária, prevalência de doenças crônicas e aumento de peso entre idosos com comprometimento cognitivo e aptidão funcional baixa, decorrente, especialmente, do sedentarismo. Em asserção ao exposto, no contexto da institucionalização, é de suma importância a avaliação física e funcional da pessoa idosa, tendo em vista que esta possibilita determinar o grau de comprometimento funcional e, sobretudo, indicar a intervenção assistencial necessária (Souza, Benedetti, Borges, Mazo, \& Gonçalves, 2011), o que justifica os resultados encontrados no presente estudo, quanto aos instrumentos utilizados para avaliação do idoso institucionalizado.

Os resultados quanto à área de conhecimento dos autores das pesquisas, foram obtidos mediante dados de filiação dos autores, disponíveis nos artigos levantados. Os dados revelam que a área do conhecimento que mais produziu pesquisas foi a enfermagem (40,50\%), seguida da fisioterapia, com $8,99 \%$ das publicações. 
Uma maior produção de estudos desenvolvidos pela área de enfermagem se justificam, uma vez que o cuidado integral do idoso, ofertado em ILPIs (tais como alimentação e higiene), e serviços à saúde (avaliação de sintomas e administração de medicação) é desempenhado por profissionais da enfermagem e cuidadores. Nessa perspectiva, Oliveira e Rozendo (2014) defendem que os enfermeiros, enquanto profissionais comprometidos com o cuidado aos seres humanos, necessitam refletir acerca de suas práticas e o contexto em que são aplicadas, com intuito em devolver aos idosos institucionalizados o papel de sujeito de suas vidas. Contudo, para que isso ocorra, faz-se necessário, investimento em pesquisa e produção de conhecimentos, que possibilitem melhoria contínua e adequação das práticas.

Cabe ressaltar, no entanto, que as ILPIs devem contar com a presença de uma equipe multiprofissional, composta por profissionais de enfermagem (enfermeiros e técnicos), médico, nutricionista, psicólogo, fisioterapeuta, educador físico, assistente social, cuidadores e profissionais de serviços gerais, com o objetivo de promover atenção integral à pessoa idosa (Silva, \& Santos, 2010). Dessa forma, a publicação insipiente envolvendo as demais áreas do conhecimento parece refletir a dificuldade das instituições de longa permanência em adequarse à legislação vigente, no que concerne à promoção de um cuidado digno, de qualidade de vida e de bem-estar aos idosos, nos domínios físico, social e psicológico. O investimento em pesquisas que abordam apenas o domínio físico (dependência e incapacidade funcional, fragilidades, morbidades e doenças), reforçam o estereótipo social da velhice como um período caracterizado por perdas e decadência, e a visão largamente difundida na sociedade de que as ILPIs são "depósitos" de velhos (Santos, et al., 2014; Salcher, Portella, \& Scortegagna, 2015).

\section{Temáticas abordadas}

A temática abordada nos estudos que tratam dos idosos, foram organizadas em categorias, a saber: (1) aspectos clínicos e nutricionais; (2) perfil sociodemográfico e epidemiológico; (3) desfechos adversos; (4) qualidade de vida e lazer; e (5) avaliação subjetiva.

A categoria referente a aspectos clínicos e nutricionais reúne artigos que abordaram sinais e sintomas apresentados pelos idosos institucionalizados. Os dados disponíveis revelam um predomínio dos estudos que buscam identificar e avaliar a farmacoterapia utilizada por idosos institucionalizados, as interações medicamentosas, o uso de medicamentos potencialmente inapropriados e a forma de administração. 
Os estudos revelam que o número de medicamentos consumidos pelas mulheres é superior, em comparação aos homens, sendo maior a frequência de consumo de psicofármacos seguidos por medicamentos cardiovasculares (Vieira de Lima, et al., 2013; Oliveira, \& Novaes, 2013; Pinto, Malaquias, Ferré, \& Pinheiro, 2013).

Prescrições inadequadas de grupos farmacológicos, dose de ingestão superior ao recomendado, medicações contraindicadas devido à presença de outras patologias, e interações medicamentosas foram reportadas em cinco estudos (Ferreira, Jerez-Roig, Ribeiro, Moreira, \& Lima, 2017; Ganassim, Matos, \& Toffoli-Kadri, 2014; Gerlack, et al., 2014; Vieira de Lima, et al., 2013; Pinto, et al., 2013).

Apenas um estudo averiguou as práticas relativas ao uso e à administração de medicações destinadas aos idosos institucionalizados e o impacto no bem-estar destes (Freitas, \& Noronha, 2013), ressaltando o cuidado necessário para que o medicamento seja destinado corretamente ao sujeito, sem erros ou trocas. Os autores destacam a importância de capacitação aos cuidadores, maior fiscalização por parte de enfermeiros, médicos e farmacêuticos, na dispensação e administração de medicamentos.

Outro tema que contempla a categoria aspectos clínicos e nutricionais, amplamente abordado pelos pesquisadores, refere-se ao estado mental e à presença de sintomas psiquiátricos. Comparativamente, os idosos institucionalizados apresentam mais declínio cognitivo e sintomas depressivos em relação aos não institucionalizados (Borges, Rocha, Couto, \& Mancini, 2013). Perdas cognitivas entre idosos institucionalizados, ajustadas segundo critérios de idade e escolaridade, também foram reportadas em outros estudos (Hartmann Junior, \& Gomes, 2016; Lampert, \& Rosso, 2015; Ferreira, Pinho, Pereira, \& Ferreira, 2014; Medeiros, Alchieri, Azevedo, Varela de Oliveira, \& Pereira, 2014).

Dos estudos levantados, apenas três analisaram os efeitos de um programa de intervenção não farmacológica no desempenho cognitivo de idosos institucionalizados (Lima Neto, Nunes, Oliveira, Azevedo, \& Mesquita,2017; Domiciano, et al., 2016; Rocha, Klein, \& Pasqualotti, 2014). Embora o declínio cognitivo seja um dos principais fatores de institucionalização de idosos, é importante destacar que a institucionalização acarreta mudanças nos padrões de vida destes, que se tornam sedentários, dependentes e são pouco estimulados, repercutindo, em longo prazo, em deterioração cognitiva.

A prevalência de incontinência urinária e os fatores associados (Melo, et al., 2017; Jerez-Roig, et al., 2016; Jerez-Roig, Santos, Souza, Amaral, \& Lima, 2016), bem como a incontinência fecal (Silva, et al., 2016), também foi objeto de investigação dos estudos 
levantados. Os autores destacam que a presença dessa condição predispõe os idosos a uma relação de dependência de cuidados contínuos, repercutindo, negativamente, na qualidade de vida, bem-estar e autoestima dos idosos. Outros três estudos levantados buscaram identificar agentes infecciosos e desordens clínicas associadas em idosos institucionalizados (Silva, \& Garbaccio, 2016; Torres, et al., 2016; Vasconcellos, et al., 2013).

O estado nutricional dos residentes em ILPIs, utilizando-se de indicadores antropométricos e exames bioquímicos foi objeto de investigação de quatro estudos (Rocha, et al., 2016; Pfrimer, et al., 2015; Pereira, et al., 2014; Volpini, \& Frangella, 2013). Outros dois estudos avaliaram o processo de alimentação, no que tange às memórias gustativas (Silva, Silva, \& Murta, 2013), e alterações das funções de mastigação e deglutição (Oliveira, Delgado, \& Brescovici, 2014).

A categoria perfil sociodemográfico e epidemiológico contempla artigos que buscaram descrever as características dos idosos institucionalizados, como sexo, estado civil, escolaridade, renda, número de filhos, número de doenças e medicamentos utilizados. Dos 12 estudos que compõem essa categoria, cinco enfocaram os fatores associados à institucionalização (Araújo, et al., 2016; Lini, et al., 2016; Pinheiro, et al., 2016; Borges, et al., 2015; Oliveira, Pavarini, Orlandi, \& Mendiondo, 2014).

$\mathrm{Na}$ categoria desfechos adversos, foram agrupados os estudos que tratam de fragilidade, capacidade funcional e quedas, decorrentes a condições pré-mórbidas. Dos estudos que perfazem esta categoria, 43,33\% (n=13), investigaram os fatores predisponentes à mobilidade reduzida e à incapacidade para atividades básicas de vida diária. Foi localizado apenas um estudo (Menezes, Aguiar, Alves, Quadros, \& Bezerra, 2016), com delineamento quase-experimental, que investigou o impacto de uma intervenção fisioterapêutica na cognição, mobilidade e capacidade funcional de idosos institucionalizados com comprometimento cognitivo leve e demência.

Os estudos que tratam de quedas (40\%), buscaram determinar a incidência e prevalência destas, bem como causas e consequências destas à saúde dos idosos institucionalizados. No que concerne aos estudos sobre fragilidade (16,67\%), destaca-se a pesquisa realizada por Santos, et al. (2017), que desenvolveram um aplicativo para dispositivos móveis, a fim de auxiliar profissionais da área da saúde, a identificar, de forma rápida e segura, características da síndrome de fragilidade.

A categoria denominada avaliação subjetiva contempla estudos que buscaram avaliar a percepção dos idosos residentes em ILPIs acerca de sua saúde, e o processo de 
institucionalização, qualidade de vida e atividades promotoras de lazer, e cuidados recebidos. Dos estudos que perfazem essa categoria $(n=14)$, destacam-se os trabalhos que compararam a qualidade de vida de idosos residentes na comunidade em relação a idosos institucionalizados (Dagios, Vasconcelos, \& Evangelista, 2015; Khoury, \& Sá-Neves, 2014).

\section{Considerações Finais}

A presente revisão integrativa permitiu verificar e analisar a produção científica acerca das ILPIs no Brasil. Embora a produção nacional acerca da referida temática tenha aumentado nos últimos anos, nota-se uma tendência entre os pesquisadores em descrever o perfil dos idosos institucionalizados, enfatizando-se aspectos sociodemográficos, epidemiológicos e clínicos. Estes achados podem decorrer da complexidade do processo de envelhecimento e da multiplicidade de fatores que repercutem em um envelhecimento bem-sucedido.

Em asserção ao exposto, ressalta-se a importância em propiciar que múltiplas áreas do saber atuem conjuntamente, tendo um olhar multidimensional acerca do envelhecimento, o que pode fomentar maior fidedignidade dos dados obtidos, bem como o desenvolvimento de políticas públicas e medidas que visem à melhoria do atendimento, prestação de serviços e qualidade de vida aos idosos institucionalizados.

\section{Referências}

Alves-Silva, J. D., Scorsolini-Comin, F., \& Santos, M. A. dos. (2013). Idosos em instituições de longa permanência: desenvolvimento, condições de vida e saúde. Psicologia: Reflexão e Crítica, 26(4), 820-830. Recuperado em 01 dezembro, 2017, de: http://www.scielo.br/pdf/prc/v26n4/23.pdf.

Araújo, A. M., Sousa Neto, T. B. d., \& Bós, Â. J. G. (2016). Differences between the profiles of institutionalized elderly people and those on waiting lists and who do not want to be institutionalized. Revista Brasileira de Geriatria e Gerontologia, 19(1), 105-118. Recuperado em 01 dezembro, 2017, de: http://dx.doi.org/10.1590/1809-9823.2016.14175.

Barros, T. V. P., Santos, A. D. B. S., Gonzaga, J. de. M. G., Lisboa, M. G. da. C. L., \& Brand, C. (2016). Capacidade funcional de idosos institucionalizados: revisão integrativa. $A B C S$ Health Sci., 41(3), 176-180. Recuperado em 01 dezembro, 2017, de: doi: https://doi.org/10.7322/abcshs.v41i3.908.

Borges, C. L., Silva, M. J. d., Clares, J. W. B., Nogueira, J. d. M., \& Freitas, M. C. d. (2015). Características sociodemográficas e clínicas de idosos institucionalizados: contribuições para o cuidado de enfermagem. Revista Enfermagem UERJ, 23(3), 7. Recuperado em 01 dezembro, 2017, de: doi: 10.12957/reuerj.2015.4214. 
Borges, M. G. de S., Rocha, L. R. da, Couto, E. de A. B., \& Mancini, P. C. (2013). Comparação do equilíbrio, depressão e cognição entre idosas institucionalizadas e nãoinstitucionalizadas. Revista CEFAC, 15(5), 1073-1079. Recuperado em 01 dezembro, 2017, de: http://www.scielo.br/pdf/rcefac/v15n5/v15n5a03.pdf.

Born, T., \& Boechat, N. S. (2013). A qualidade dos cuidados ao idoso institucionalizado. In: E. V. Freitas, L. Py, \& J. Doll. (Eds.). Tratado de Geriatria e Gerontologia (3 ${ }^{\mathrm{a}}$ ed., pp. 18201835). Rio de Janeiro, RJ: Guanabara Koogan.

Brasil. (1989). Portaria n. ${ }^{\circ}$ 810, de 22 de setembro de 1989. Brasília, DF: Ministério da Saúde

Brasil. (2005). Regulamento Técnico que define normas de funcionamento para as Instituições de Longa Permanência para Idosos.

Bruinsma, J. L., Beuter, M., Leite, M. T., Hildebrandt, L. M., Venturini, L., \& Nishijima, R. B. (2017). Conflitos entre idosas institucionalizadas: dificuldades vivenciadas pelos profissionais de enfermagem. Escola Anna Nery, 21(1), e20170020. Recuperado em 01 dezembro, 2017, de: file:///C:/Users/Dados/Downloads/1414-8145-ean-21-01-e20170020.pdf.

Camarano, A. A., \& Kanso, S. (2010). As instituições de longa permanência para idosos no Brasil. Revista Brasileira de Estudos de População, 27(1) 232-235. Recuperado em 01 dezembro, 2017, de: http://www.scielo.br/pdf/rbepop/v27n1/14.pdf,

Clos, M. B., \& Grossi, P. K. (2016). Desafios para o cuidado digno em instituições de longa permanência. Revista Bioética, 24(2), 395-411. Recuperado em 01 dezembro, 2017, de: http://dx.doi.org/10.1590/1983-80422016242140., 395-411.

Comiotto, G. S., Kappaun, S., \& Cesa, C. C. (2016). Conhecimento dos profissionais da área da saúde acerca da comunicação suplementar e alternativa em instituições de longa permanência para idosos. Revista CEFAC, 18(5), 1161-1168. Recuperado em 01 dezembro, 2017, de: http://dx.doi.org/10.1590/1982-0216201618522215.

Dagios, P., Vasconcellos, C., \& Evangelista, D. H. R. (2015). Avaliação da qualidade de vida: comparação entre idosos não institucionalizados participantes de um centro de convivência e idosos institucionalizados em JI-Paraná/RO. Estudos Interdisciplinares sobre o Envelhecimento, 20(2), 469-484. Recuperado em 01 dezembro, 2017, de: https://seer.ufrgs.br/RevEnvelhecer/article/view/41571.

Domiciano, B. R., Braga, D. K. A. P., Silva, P. N. d., Santos, M. P. A. d., Vasconcelos, T. B. d., \& Macena, R. H. M. (2016). Cognitive function of elderly residents in long-term institutions: effects of a physiotherapy program. Revista Brasileira de Geriatria $e$ Gerontologia, 19(1), 57-70. Recuperado em 01 dezembro, 2017, de: http://dx.doi.org/10.1590/1809-9823.2016.14137.

Ferreira, L. M. d. B. M., Jerez-Roig, J., Ribeiro, K. M. O. B. d. F., Moreira, F. S. M., \& Lima, K. C. d. (2017). Associação entre medicamentos de uso contínuo e tontura em idosos institucionalizados. Revista CEFAC, 19(3), 381-386. Recuperado em 01 dezembro, 2017, de: http://www.scielo.br/pdf/rcefac/v19n3/1982-0216-rcefac-19-03-00381.pdf.

Ferreira, L. S., Pinho, M. do S. P., Pereira, M. W. de M., \& Ferreira, A. P. (2014). Perfil cognitivo de idosos residentes em Instituições de Longa Permanência de Brasília, DF. Revista Brasileira de Enfermagem, 67(2), 247-251. Recuperado em 01 dezembro, 2017, de: http://dx.doi.org/10.5935/0034-7167.20140033. 
Freitas, A. V. da S., \& Noronha, C. V. (2013). Uso de medicamentos em idosos que residem em uma instituição de longa permanência. Estudos Interdisciplinares sobre o Envelhecimento, 18(1), 105-118. Recuperado em 01 dezembro, 2017, de: https://seer.ufrgs.br/RevEnvelhecer/article/view/19815.

Ganassin, A. R., Matos, V. T. G. de, \& Toffoli-Kadri, M. C. (2014). Potentially inappropriate medication use in institutionalized older adults according to the Beers Criteria. Brazilian Journal of Pharmaceutical Sciences, 50(4), 827-837. Recuperado em 01 dezembro, 2017, de: http://www.scielo.br/pdf/bjps/v50n4/1984-8250-bjps-50-04-00827.pdf.

Gerlack, L. F., Cuentro, V. de S., Estrela, M. F. B., Karnikowski, M. G. de O., Pinho, D. L. M., \& Bós, Â. J. G. (2014). Interações medicamentosas na farmacoterapia prescrita a idosos residentes em uma instituição de longa permanência brasileira. Estudos Interdisciplinares sobre o Envelhecimento, 19(2), 439-452. Recuperado em 01 dezembro, 2017, de: https://seer.ufrgs.br/RevEnvelhecer/article/view/43273.

Hartmann Junior, J. A. S., \& Gomes, G. C. (2016). Depressão em Idosos Institucionalizados: Padrões Cognitivos e Qualidade de Vida. Ciências \& Cognição, 21(1), 137-154. Recuperado em 01 dezembro, 2017, de: http://www.cienciasecognicao.org/revista/index.php/cec/ article/view/1028.

Jerez-Roig, J., Santos, M. M., Souza, D. L. B., Amaral, F. L. J. S., \& Lima, K. C. (2016). Prevalence of urinaryincontinence and associated factors in nursing home residents. Neurourology and Urodynamics, 35(1), 102-107. Recuperado em 01 dezembro, 2017, de: doi: 10.1002/nau.22675.

Jerez-Roig, J., Souza, D. L. B., Andrade, F. L. J. P. de, Lima Filho, B. F. de, Medeiros, R. J. de, Oliveira, N. P. D. de, Cabral Neto, S. M., \& Lima, K. C. (2016). Autopercepção da saúde em idosos institucionalizados. Ciência \& Saúde Coletiva, 21(11), 3367-3375. Recuperado em 01 dezembro, 2017, de: http://dx.doi.org/10.1590/1413-812320152111.15562015.

Khoury, H. T. T., \& Sá-Neves, Â. C. (2014). Percepção de controle e qualidade de vida: comparação entre idosos institucionalizados e não institucionalizados. Revista Brasileira de Geriatria e Gerontologia, 17(3), 553-565. Recuperado em 01 dezembro, 2017, de: http://dx.doi.org/10.1590/1809-9823.2014.13012.

Lampert, M. A., \& Rosso, A. L. P. (2015). Depression in elderly women resident in a longstay nursing home. Dementia \& Neuropsychologia, 9(1), 76-80. Recuperado em 01 dezembro, 2017, de: http://www.demneuropsy.com.br/detalhe_artigo.asp?id=506.

Lima Neto, A. V. d. L., Nunes, V. M. d. A., Oliveira, K. S. A. d., Azevedo, L. M. d., \& Mesquita, G. X. B. (2017). Estimulação em idosos institucionalizados: efeitos da prática de atividades cognitivas. Revista de Pesquisa: Cuidado é Fundamental Online, 9(3), 753-759. Recuperado em 01 dezembro, 2017, de: http://www.seer.unirio.br/index.php/ cuidadofundamental/article/view/5491.

Lini, E. V., Portella, M. R., \& Doring, M. (2016). Factors associated with the institutionalization of the elderly: a case-control study. Revista Brasileira de Geriatria $e$ Gerontologia, 19(6), 1004-1014. Recuperado em 01 dezembro, 2017, de: http://dx.doi.org/10.1590/1981-22562016019.160043.

Mariano, P. P., Baldissera, V. D. A., Martins, J. T., \& Carreira, L. (2015). Nursing work organization in long-stay institutions for the elderly: relationship to pleasure and suffering at work. Texto \& Contexto - Enfermagem, 24(3), 756-765. Recuperado em 01 dezembro, 2017, de: http://dx.doi.org/10.1590/0104-070720150-1150014. 
Mariano, P. P., \& Carreira, L. (2016a). Estratégias defensivas no ambiente laboral da enfermagem nas instituições de longa permanência para idosos. Revista Gaúcha de Enfermagem, 37(3), e58587. Recuperado em 01 dezembro, 2017, de: http://www.scielo.br/pdf/rgenf/v37n3/0102-6933-rgenf-1983-144720160358587.pdf.

Mariano, P. P., \& Carreira, L. (2016b). Prazer e sofrimento no cuidado ao idoso em instituição de longa permanência: percepção dos trabalhadores de enfermagem. Escola Anna Nery, 20(4), e20160088. Recuperado em 01 dezembro, 2017, de: http://dx.doi.org/10.5935/1414-8145.20160088.

Medeiros, A. N. V. d., Alchieri, J. C., Azevedo, L. M., Varela de Oliveira, K. M., \& Pereira, D. A. (2014). Cognitive Assessment in Elderly Residents of Long-Stay Institutions. Dementia and Geriatric Cognitive Disorders, 37(1-2), 27-33. Recuperado em 01 dezembro, 2017, de: https://doi.org/10.1159/000348348.

Medeiros, F. d. A. L., Félix, L. G., \& Nóbrega, M. M. L. de. (2016). Processo Clinical Caritas em oficinas para cuidadores de idosos institucionalizados. Revista Brasileira de Enfermagem, 69(6), 997-1004. Recuperado em 01 dezembro, 2017, de: doi: http://dx.doi.org/ 10.1590/0034-7167-2016-0359.

Medeiros, F. de A. L., Oliveira, J. M. M., Lima, R. J. de, \& Nobrega, M. M. L. de. (2015). O cuidar de pessoas idosas institucionalizadas na percepção da equipe de enfermagem. Revista Gaúcha de Enfermagem, 36(1), 56-61. Recuperado em 01 dezembro, 2017, de: https://seer.ufrgs.br/RevistaGauchadeEnfermagem/article/view/45636.

Melo, L. S. d., Ercole, F. F., Oliveira, D. U. d., Pinto, T. S., Victoriano, M. A., \& Alcoforado, C. L. G. C. (2017). Urinary tract infection: a cohort of older people with urinary incontinence. Revista Brasileira de Enfermagem, 70(4), 838-844. Recuperado em 01 dezembro, 2017, de: http://www.scielo.br/pdf/reben/v70n4/pt_0034-7167-reben-70-04-0838.pdf.

Menezes, A. V., Aguiar, A. d. S. d., Alves, E. F., Quadros, L. B. d., \& Bezerra, P. P. (2016). Efetividade de uma intervenção fisioterapêutica cognitivo-motora em idosos institucionalizados com comprometimento cognitivo leve e demência leve. Ciência \& Saúde Coletiva, 21(11), 3459-3467. Recuperado em 01 dezembro, 2017, de: http://dx.doi.org/10.1590/1413-812320152111.17892015.

Negreiros, N. P. V. d., Cavalcanti, M. Á. C., Marques, d. A. H. C., Ricardo, O. G., \& Fontenele, A. J. (2014). Sleep and frailtysyndrome in elderly residents of long-stay institutions: A cross-sectional study. Geriatrics \& Gerontology International, 14(3), 605-612. Recuperado em 01 dezembro, 2017, de: doi: 10.1111/ggi.12144.

Nunes, J. T., Nunes, J. T., Marinho, A. C. V., \& Fernandes, M. N. d. F. (2014). Reflexões sobre os cuidados de enfermagem a idosos institucionalizados. São Paulo, SP: PUC-SP: Revista Kairós-Gerontologia, 17(1), 355-373. Recuperado em 01 dezembro, 2017, de: file://C:/Users/Dados/Downloads/21390-54780-1-SM.pdf.

Oliveira, B. S. d., Delgado, S. E., \& Brescovici, S. M. (2014). Alterações das funções de mastigação e deglutição no processo de alimentação de idosos institucionalizados. Revista Brasileira de Geriatria e Gerontologia, 17(3), 575-587. Recuperado em 01 dezembro, 2017, de: http://dx.doi.org/10.1590/1809-9823.2014.13065.

Oliveira, J. M. de, \& Rozendo, C. A. (2014). Instituição de longa permanência para idosos: um lugar de cuidado para quem não tem opção? Revista Brasileira de Enfermagem, 67(5), 773-779. Recuperado em 01 dezembro, 2017, de: http://dx.doi.org/10.1590/00347167.2014670515 . 
Oliveira, M. P. F. d., \& Novaes, M. R. C. G. (2013). Perfil socioeconômico, epidemiológico e farmacoterapêutico de idosos institucionalizados de Brasília, Brasil. Ciência \& Saúde Coletiva, 18(4), 1069-1078. Recuperado em 01 dezembro, 2017, de: http://dx.doi.org/10.1590/S1413-81232013000400020.

Oliveira, S. C. d., Pavarini, S. C. I., Orlandi, F. d. S., \&Mendiondo, M. S. Z. d. (2014). Family functionality: A study of Brazilian institutionalized elderly individuals. Archives of Gerontology and Geriatrics, 58(1), 170-176. Recuperado em 01 dezembro, 2017, de: doi: https://doi.org/10.1016/j.archger.2013.07.003.

Pereira, M. L. A. S., Moreira, P. d. A., Oliveira, C. C. d., Roriz, A. K. C., Amaral, M. T. R., Mello, A. L., \& Ramos, L. B. (2014). Nutritional status of institutionalized elderly Brazilians: a study with the Mini Nutritional Assessment. Nutr Hosp., 31(3), 1198-1204. Recuperado em 01 dezembro, 2017, de: doi: 10.3305/nh.2015.31.3.8070.

Pestana, L. C., \& Espírito Santo, F. H. d. (2008). As engrenagens da saúde na terceira idade: um estudo com idosos asilados. Revista da Escola de Enfermagem da USP, 42(2), 268-275. Recuperado em 01 dezembro, 2017, de: http://cacphp.unioeste.br/extensao/unati/arqs/UNATI_08.pdf.

Pfrimer, K., Marques Messias, M., Ferriolli, E., Vieira Salles, M. S., Roma Junior, L. C., Saran Netto, A., Zanetti, M. A., Vannucchi, H. (2015). Avaliação e acompanhamento nutricional em idosos de uma instituição de longa permanência. Arch. Latinoam. Nutr, 65(2), 104-109. Recuperado em 01 dezembro, 2017, de: http://bases.bireme.br/cgibin/wxislind.exe/iah/online/?IsisScript=iah/iah.xis\&src=google \&base=LILACS\&lang=p\&nex tAction=lnk\&exprSearch=752720\&indexSearch=ID.

Pinheiro, N. C. G., Holanda, V. C. D., Melo, L. A. d., Medeiros, A. K. B. d., \& Lima, K. C. d. (2016). Desigualdade no perfil dos idosos institucionalizados na cidade de Natal, Brasil. Ciência \& Saúde Coletiva, 21(11), 3399-3405. Recuperado em 01 dezembro, 2017, de: http://dx.doi.org/10.1590/1413-812320152111.19472015.

Pinto, M. C. X., Malaquias, D. P., Ferré, F., \& Pinheiro, M. L. P. (2013). Potentially inappropriate medication use among institutionalized elderly individuals in South eastern Brazil. Brazilian Journal of Pharmaceutical Sciences, 49(4), 709-717. Recuperado em 01 dezembro, 2017, de: http://dx.doi.org/10.1590/S1984-82502013000400010.

Rocha, J. d. P., Klein, O. J., \& Pasqualotti, A. (2014). Qualidade de vida, depressão e cognição a partir da educação gerontológica mediada por uma rádio-poste em instituições de longa permanência para idosos. Revista Brasileira de Geriatria e Gerontologia, 17(1), 115128. Recuperado em 01 dezembro, 2017, de: http://www.scielo.br/pdf/rbgg/v17n1/18099823-rbgg-17-01-00115.pdf.

Rocha, É. C. P. F., Mello, C. Y. S. V. d., Moreira, M. d. A., Ferreira, E. A., Ogava, T. S., \& Carvalho, E. E. M. C. d. (2016). Perfil nutricional de idosas residentes em instituição de longa permanência da cidade do Recife, PE, a partir de diferentes métodos antropométricos. Nutrición clínica y dietética hospitalaria, 36(2), 38-43. Recuperado em 01 dezembro, 2017, de: https://revista.nutricion.org/PDF/forterocha.pdf.

Roquete, F. F., Batista, C. C. R. F., \& Arantes, R. C. (2017). Care and management demands of long-term care facilities for the elderly in Brazil: na integrative review (2004-2014). Revista Brasileira de Geriatria e Gerontologia, 20(2), 286-299. Recuperado em 01 dezembro, 2017, de: http://dx.doi.org/10.1590/1981-22562017020.160053. 
Salcher, E. B. G., Portella, M. R., \& Scortegagna, H. d. M. (2015). Cenários de instituições de longa permanência para idosos: retratos da realidade vivenciada por equipe multiprofissional. Revista Brasileira de Geriatria e Gerontologia, 18(2), 259-272. Recuperado em 01 dezembro, 2017, de: http://dx.doi.org/10.1590/1809-9823.2015.14073.

Santos, N. O. dos, Beuter, M., Girardon-Perlini, N. M. O., Paskulin, L. M. G., Leite, M. T., \& Budó, M. de L. D. (2014). Percepção de trabalhadores de uma instituição de longa permanência para idosos acerca da família. Texto Contexto Enfermagem, 23(4), 971-978. Recuperado em 01 dezembro, 2017, de: http://dx.doi.org/10.1590/010407072014003170013.

Santos, T. S. dos, Brito, T. A., Yokoyama Filho, F. S., Guimarães, L. de A., Souto, C. S., Souza, S. J. N., Martins, L. E. B., \& Pithon, K. R. (2017). Development of na application for mobile devices to identify the frailty phenotype among the elderly. Revista Brasileira de Geriatria e Gerontologia, 20(1), 67-73. Recuperado em 01 dezembro, 2017, de: http://dx.doi.org/10.1590/1981-22562017020.160025.

Scarabottolo, C. C., Garcia Júnior, J. R., Gobbo, L. A., Alves, M. J., Ferreira, A. D., Zanuto, E. A. C., Oliveira, W. G. A. de, \& Christofaro, D. G. D. (2017). Influence of physical exercise on the functional capacity in institutionalized elderly. Revista Brasileira de Medicina do Esporte, 23(3), 200-203. Recuperado em 01 dezembro, 2017, de: http://dx.doi.org/10.1590/1517-869220172303150175.

Silva, A. G. S., \& Garbaccio, J. L. (2016). Registro do uso de antimicrobianos em Instituições de Longa Permanência para Idosos. Revista Brasileira de Geriatria e Gerontologia, 19(2), 325-334. Recuperado em 01 dezembro, 2017, de: http://dx.doi.org/10.1590/180998232016019.150032 .

Silva, B. T. da, \& Santos, S. S. C. (2010). Cuidados aos idosos institucionalizados: opiniões do sujeito coletivo enfermeiro para 2026. Acta Paulista de Enfermagem, 23(6), 775-781. Recuperado em 01 dezembro, 2017, de: http://dx.doi.org/10.1590/S010321002010000600010.

Silva, K. A., Silva, M. d. F. G., \& Murta, N. M. G. (2013). Práticas alimentares e bem-estar de residentes de uma Instituição de Longa Permanência para Idosos da cidade de Diamantina, MG, Brasil. São Paulo, SP: PUC-SP: Revista Kairós-Gerontologia, 16(3), 221-236. Recuperado em 01 dezembro, 2017, de: file:///C:/Users/Dados/Downloads/27564-72416-1SM\%20(3).pdf.

Silva, M. A., Aguiar, E. S. S., Matos, S. D. d. O., Lima, J. d. O., Costa, M. M. L., \& Soares, M. J. G. O. (2016). Prevalência de incontinência urinária e fecal em idosos: estudo em instituições de longa permanência para idosos. Estudos Interdisciplinares sobre o Envelhecimento, 21(1), 249-261. Recuperado em 01 dezembro, 2017, de: https://seer.ufrgs.br/RevEnvelhecer/article/view/46484.

Silva, R. J. M. d., Dias, S. M. S., \& Piazza, L. (2017). Desempenho em atividades de simples e dupla tarefas de idosos institucionalizados que realizam e não realizam fisioterapia. Fisioterapia e Pesquisa, 24(2), 149-156. Recuperado em 01 dezembro, 2017, de: http://dx.doi.org/10.1590/1809-2950/16610424022017.

Souza, P. D. d., Benedetti, T. R. B., Borges, L. J., Mazo, G. Z., \& Gonçalves, L. H. T. (2011). Aptidão funcional de idosos residentes em uma instituição de longa permanência. Revista Brasileira de Geriatria e Gerontologia, 14(1) 07-16. Recuperado em 01 dezembro, 2017, de: http://dx.doi.org/10.1590/S1809-98232011000100002. 
Torres, J. D. P. R. V., Nobre, S. A. M., Silva, J. R., Caldeira, M. T. G., Silva, T. N., Torres, S. d. A. S., \& Andrade, M. C. (2016). Microbiota intestinal e associações com desordens clínicas em função da faixa etária de idosos: um estudo analítico transversal. Estudos Interdisciplinares sobre o Envelhecimento, 21(1), 263-281. Recuperado em 01 dezembro, 2017, de: https://seer.ufrgs.br/RevEnvelhecer/article/view/59125.

Trindade, A. P. N. T. d., Barboza, M. A., Oliveira, F. B. d., \& Borges, A. P. O. (2013). Repercussão do declínio cognitivo na capacidade funcional em idosos institucionalizados e não institucionalizados. Fisioterapia em Movimento, 26(2), 281-289. Recuperado em 01 dezembro, 2017, de: http://dx.doi.org/10.1590/S0103-51502013000200005.

Vasconcellos, C., Pereira, C. Q. M., Souza, M. C., Pelegrini, A., Freitas, R. S., \& Takahashi, J. P. (2013). Identification of fungi species in the on ychomycosis of institutionalized elderly. Anais Brasileiros de Dermatologia, 88(3), 377-380. Recuperado em 01 dezembro, 2017, de: http://dx.doi.org/10.1590/abd1806-4841.20131884.

Vieira de Lima, T. J., Garbin, C. A. S., Garbin, A. J. Í., Sumida, D. H., \& Saliba, O. (2013). Potentially inappropriate medications used by the elderly: prevalence and risk factors in Brazilian care homes. BMC Geriatrics, 13(1), 52. Recuperado em 01 dezembro, 2017, de: doi: 10.1186/1471-2318-13-52.

Volpini, M. M., \& Frangella, V. S. (2013). Avaliação nutricional de idosos institucionalizados. Einstein (São Paulo), 11(1) 32-40. Recuperado em 01 dezembro, 2017, de: http://dx.doi.org/10.1590/S1679-45082013000100007.

Whittemore, R., \& Knafl, K. (2005). The integrative review: updated methodology. Journal of Advanced Nursing, 52(5), 546-553. Recuperado em 01 dezembro, 2017, de: doi: 10.1111/j.1365-2648.2005.03621.x.

Recebido em 20/07/2018

Aceito em 30/09/2018

Patricia do Nascimento Tavares - Mestrado em Ciências do Envelhecimento, Universidade

São Judas Tadeu. Professora assistente do Centro Universitário das Faculdades

Metropolitanas Unidas. Doutoranda em Gerontologia, Universidade Estadual de Campinas.

E-mail: patricia_ntavares@hotmail.com 
Olga Susana Costa Couto e Araújo - Doutoranda em Gerontologia, UNICAMP. Mestre em Museologia, USP. Licenciatura em Antropologia, Universidade Nova de Lisboa. Pósgraduação em Envelhecimento e autonomia funcional na Universidade Técnica de Lisboa (FMH), Portugal.

E-mail: susanacostaaraujo@gmail.com

Josiane Ferreira de Mello - Graduação em Nutrição. Docente no Centro Universitário de Jaguariúna (UNIFAJ), no Serviço Nacional de Aprendizagem Comercial (SENAC), e Nutricionista clínica, com experiência em Nutrição do idoso. Mestre em Gerontologia, Universidade Estadual de Campinas, UNICAMP.

E-mail: josianefmello@yahoo.com.br

Andreza de Nóvoa Rocha Maciel - Graduada em Fisioterapia, Universidade Paulista e Licenciatura plena em Educação Física, Universidade do Estado do Pará. Pós-Graduada em Fisioterapia aplicada à Traumatologia, Unicamp. Mestranda em Gerontologia, Unicamp. E-mail: dezanovoa@gmail.com

Maria José D'Elboux - Graduação em Enfermagem, PUC-SP. Mestrado e Doutorado em Enfermagem, Universidade de São Paulo. Livre Docência, Universidade Estadual de Campinas. Atualmente é professor colaborador na Universidade Estadual de Campinas, no Programa de Pós-Graduação em Gerontologia da Faculdade de Ciências Médicas.

E-mail: mjdelboux@gmail.com 